\title{
FEED - Modeling the relationship between education and food waste
}

\author{
Valentina Mihaela GHINEA \\ Bucharest University of Economic Studies, Bucharest, Romania \\ valentina_ghinea@yahoo.com \\ Ramona Elena CANTARAGIU \\ Bucharest University of Economic Studies, Bucharest, Romania \\ ramona_cantaragiu@yahoo.com \\ Mihalache GHINEA \\ University POLITEHNICA of Bucharest, Bucharest, Romania \\ ghinea2003@yahoo.com
}

\begin{abstract}
As formed by humans, which are living creatures full of contradictions, our society is characterized as well by lots of paradoxes. One could say that it has never been so wealthy and educated, while others would declare themselves as being grateful for a simple glass of water or a slice of bread, as the world wide abundance of goods and opportunities is counterpointed by deep scarcity, sometimes not too far from the sources of waste. Therefore, quite large amounts of edible food that could have been consumed end up in landfills, thus contributing to environmental pollution and social disparities. Despite many studies conducted in order to better understand the causes of this phenomenon, and although at the EU and UN level some actions were taken in order to reduce consumer food waste, the topic still remains open and it lacks a clear and impactful approach. In this light, we made use of the results of previous studies, and we built the causal model, FEED, based on system dynamics, with the aim to explore the impact of the evolution of educational attainment on the aggregate of household food waste. We then translated the model into the tenet of the dynamic simulation software, TRUE. There was no reinforcing loop displayed by FEED causal loop version, fact aligned with the evolution of our goal-variable when the simulation of the model was run, a result that make us to suspect the possibility of reducing food waste in the foreseeable future.
\end{abstract}

Keywords: higher education, consumer food waste, causal loop, dynamic simulation, dynamic model.

\section{Introduction}

Food waste represents the amount of edible food that could have been consumed by individuals but instead ends up in landfills, thus contributing to environmental pollution, the ineffective use of resources and social disparities (Richter, 2017). At the level of the European Union, there is a clear desire to reduce the amount of food wasted as made evident by the 2012 resolution signed by the European Parliament, and to halve the amount of food waste by 2025 (European Parliament, 2012). The food waste reduction is also one of the sustainable development goals established by the United Nations as targets for 2030. Unfortunately, households food waste continues to represent the largest proportion of food waste generated in the entire food chain at global and European level (Stancu, Haugaard \& Lähteenmäki., 2016; Principato, Secondi \& Pratesi, 2015), in spite of various awareness campaigns having been conducted to make individuals aware of the problems generated by food waste and to provide them with instructions and incentives to adopt food related behaviors that reduce waste (Jörissen et al., 2015). In light of this, it is evident that further research is necessary in order to understand the factors that affect food waste at the consumer level and to be able to design more effective strategies aimed at the reduction of household waste to secure the attainment of the food waste goals established by the EU and the UN in the next 10 years. 
The aim of the present study is to join the debate on the influence of socio-demographic factors on the amount of food waste generated at consumer levels. Previous studies have shown that gender, age, level of income and household composition have a significant impact on household food waste (Stancu et al., 2016; Secondi et al., 2015; Neff, Spiker \& Truant, 2015; Pakpour et al., 2014; Koivupuro et al., 2012), whereas the influence of education has been only sparsely discussed (Porpino, 2016).

PICBE | 756

Based on a review of the literature, a set of five factors were found to influence the direct impact of the level of education and the amount of consumer food waste: food waste attitudes, food waste behaviors, educational campaigns, consumerism and non-consumer related factors that support the prevention of food waste (i.e., legislation, facilities, technological innovation etc.). Using the results of previous studies, we have built a causal model based on system dynamics with the aim to explore the impact of the evolution of educational attainment on the aggregate of household food waste.

\section{The Impact of Education on Food Waste}

The impact of the level of education on food waste at the consumer level has been sparingly investigated in the literature, with mixed results. Some studies have found no significant correlation between the level of education and the amount of food wasted by households (Koivupuro et al., 2012; Neff et al., 2015), while other studies have found that households with a higher level of education waste less food than households with a lower level of education (Abdelradi, 2018). However, most studies agree that a higher level of education is correlated with an increase in the amount of food wasted at the household level (Visschers et al., 2016; Secondi et al., 2015). In particular, the study performed by Secondi et al. (2015) based on data from Eurostat on EU-27 countries demonstrated that people who left school at the age of 15 or earlier are more likely to waste less than $5 \%$ of the food they bought in comparison to people who left school at the age of 20 or later. A potential explanation for the positive relationship between level of education and food waste is related to the fact that most households with a higher level of education also have higher incomes that have been shown to be linked to higher levels of food waste (Porpino, 2016). Contradictorily, the level of education is also correlated with more positive attitudes towards the prevention of food waste and with a higher adoption of behaviors aimed at reducing food waste in the household. This complicates the relationship between the level of education and the amount of food waste generated by households, thus meriting a closer exploration through the means of modelling.

Several studies have shown that the level of education has an impact on the individuals' attitudes towards food waste. For example, Qi and Roe (2016) found that individuals with higher levels of education were more likely to agree that food waste has a negative impact on the environment and that bulk purchases are related to more food waste at the household level. In addition, Djekic et al. (2019) found that individuals with higher levels of education were more likely to fit into the cluster of "caring food wasters" who feel guilty for wasting food and associate food waste with social issues such as world hunger. In contrast, Neff et al. (2015) found no significant impact of the level of education on consumers' attitudes towards food waste. In light of these studies, it can be concluded that the level of education has a moderate effect on the individuals' attitudes towards food waste, and that there are other socio-demographic variables such as age and income, which affect attitudes towards food waste to a larger extent (Melbye, Onozaka \& Hansen, 2016). 
According to the theory of planned behavior, attitudes play an important role in shaping individual's behaviors in regards to food waste. Graham-Rover, Jessop and Sparks (2015) found that the intention to reduce household food waste is predicted by attitude, subjective norms and perceived behavioral control, but no significant influence of the level of education is exerted on the dependent variables. Similarly, Karim Ghani et al. (2013) found that the level of education had a significant impact only on situational factors such as not having enough space to recycle and separate leftover food and not having enough time for food recycling, and not on attitudes, norms or behaviors related to food recycling.

In addition, a study performed based on the Dataset Flash Eurobarometer 425 and published by the European Commission, showed that the level of education had a direct positive influence on food waste related behaviors only for Western and Eastern countries, but not for Mediterranean countries. It also concluded that education had an indirect positive influence on food waste related behaviors through date label checking and understanding (Toma, Font \& Thompson, 2020). Surprisingly, Mallinson, Russell and Barker (2016) found that individuals with higher levels of education are most likely to fit into the cluster of "epicures" or "traditional consumers" rather than into the cluster of "kitchen evaders". This would mean that higher education is associated with a high degree of self-efficacy related to cooking, the allocation of time for proper cooking and high concerns related to the amount of food wasted. Based on these findings, it is evident that the level of education is positively related with behaviors that reduce the amount of food wasted at the consumer level.

In terms of the influence of cultural factors, Aschemann-Witzel et al. (2015) discussed the impact of the consumerist culture and abundance on the propensity of individuals to buy more food than necessary and to be less concerned about the negative impact of food waste. Moreover, the social norms which exist in many cultures regarding the "good mother" who has to secure food for her family or regarding the "purity" of the household have a tendency to increase the amount of food wasted and to discourage individuals from recycling leftover food (Porpino, 2016; Visschers et al., 2016). In addition, Stefan et al. (2013) found that food waste behaviors are less visible in comparison to other waste behaviors such as the amount of recycling and concluded that pro-social norms related to food waste have only a small influence on individual's behaviors. Even though studies consistently show that a higher level of education is related to a higher propensity towards anti-consumerist values, the same studies show that there is a clear gap between attitudes and behaviors and that, in most cases, anti-consumerist attitudes do not translate into sustainable consumption practices (Newton, 2013; Nässén, 2017). Thus, it can be surmised that cultural factors act as one of the drivers of food waste, irrespective of the level of education of the individual.

Educational campaigns are said to be useful in diminishing the negative influence of cultural factors on food waste, but the results from the available studies are relatively mixed as well. For example, educational campaigns aimed at changing individuals' attitudes towards food waste by making them feel bad about wasting food are rarely effective in contrast to campaigns aimed at giving individuals' tools useful for changing planning and shopping behaviors (Stefan et al., 2013; Abeliotis, Lasaridi \& Chroni, 2014). This is supported by the analysis of the outcomes generated by the "Love food hate waste" campaign launched in the UK in 2007 which led to the reduction of food waste at household levels by approximately $15 \%$ through its emphasis on increasing people's food skills using face-to-face trainings (Yamakawa et al., 2017). In addition, Young et al. (2017) found that social media food waste campaigns had no effect on individuals' food related behaviors and concluded that educational campaigns are only useful when they have a face-to-face component. Thus, educational campaigns can contribute to the reduction of food 
waste at the consumer level, but we have to bear in mind that most of these campaigns are done sparingly and that not all of them are designed in the most effective manner.

Lastly, there are also non-consumer related factors that help prevent food waste at household level. For example, the existence of a clear legislative framework related to waste recycling can help motivate individuals to adopt such behaviors and thus reduce the amount of food that ends up in landfills (Pakpour et al., 2014). Additionally, legislation that clarifies the labelling used for food products is also useful in helping people consume food products before their expiration date and thus reduce food waste (Lusk \& Ellison, 2016). Also, the implementation of pay as you throw schemes can improve individuals' awareness of the amount of food waste generated and also create economic incentives to reduce food waste (Gaiani et al., 2018; Lusk \& Ellison, 2016). Technological innovations such as smart carts that help with planning, smart packaging that signals when the food is close to the expiration date or smart refrigerators that monitor the level of food consumption can also contribute to the reduction of food waste (Gaiani et al., 2018). Changes in the level of food prices that result from periods of scarcity of raw materials are also conducive to reduction of food waste at the household level (Aschemann-Witzel et al., 2015). Overall, these factors have a significantly negative impact on the amount of food wasted at the consumer level and it is estimated that their influence will increase as more countries join the efforts towards zero food waste in the following years.

\section{Complex Systems Modelling and Simulation}

A thorough analysis of a system formed of interconnections requires the possibility to continuously interrogate the evolution of the system by expanding and compressing the time spam, and also looking at the system's evolution backwards and forwards in time (Sadjan \& Taghirad, 2005). As a result, simulations represent useful tools that allow researchers to look at the potential realities and that can be easily and cost-effectively manipulated to anticipate potential evolutions of specific social phenomena.

A couple of decades ago, simulation was rather difficult because it required highly specialized knowledge and computer programming skills. In spite of this, there were quite a few software solutions on the market such as Cellular Autonoma, used in mathematics, physics, complexity science and biology, Genetic Algorithm, that imitated the natural evolutionary processes and could be used also for optimization problems, PowerSim, ithink and STELLA, based on the programming language created by Jay Forrester and his MIT team. The aim of these software was rather similar, namely to facilitate the dynamic simulation of the model under investigation and to provide a more user-friendly tool to people with less knowledge of mathematics and computer programming as is the case with most managers and other decision-makers (Hannon \& Ruth, 2001; Sterman, 2002). In addition, according to Hanneman (1988), when it comes to the understanding of the behavior of (social) complex systems, the use of mathematical representation can either oversimplify or overcomplicate the model in comparison to the real situation. Reversely, a dynamic simulation is focused on the interaction of the system parts, be it linear and non-linear altogether, so that their inter-influenced evolution is better captured and forecasted.

There are quite a few skeptical scientists who fear that the dynamic systems approach lacks rigor, and thus argue in favor of complex non-linear systems based on mathematics to the detriment of Peter Senge's (1990) system dynamics approach, which is not based on mathematics at all. Moreover, there are also scientists and modelers who reject the utilization of soft variables (i.e., non-numerical data) despite their importance in the model and simply restrict their models to 
parameters that can be mathematically estimated. Unfortunately, the excuse according to which soft variables and relationships cannot be accurately estimated does not save or improve the reliability or relevancy of the model as Forrester (1961, p. 57) said: " to omit such variables is equivalent to saying they have zero effect - probably the only value that is known to be wrong!". Besides, as Sterman (2002) argued, what in the past was considered a soft variable (i.e., political sentiments, depression, wellbeing etc.), is now routinely measured and quantified through surveys, content analysis and other tools, which proves that it is best for models to include all relevant variables, irrespective of their nature.

Basically, everything comes down to real life reinforcing or balancing feedback loops that always reengage the system, which is rather impossible to capture with a simple linear sequence of steps as in statistics and econometrics. Even though it may seem strange, non-linearity, time delays and feedback loops are counterintuitive (Forrester, 1999) and therefore difficult to understand, perceive and anticipate. Thus, we need a formal model and its dynamic simulation to test our own mental models and our intuition. Unfortunately, there is no guarantee that the model simulation will be accurate or that the forecasting will prove true, because, as George Box used to say, "essentially, all models are wrong, but some are useful" (Box, 1987, p. 424).

\section{Research Methodology}

The construction of the model that expresses the relationship between education and food waste began with a thorough review of the literature. More than 30 articles and research reports were reviewed and helped with the identification of six compounded soft variables liable to influence the rise and fall of the levels of food wasted at household level: consumer-culture, educational campaigns, non-consumer related factors that help prevent food waste, attitudes regarding food waste, behaviors regarding food waste and higher education graduates.

We then made use of causal loops diagrams, a method popularized by Kimm (1993) and Senge (1999), emphasizing the interconnectivity existent between the above-mentioned variables and therefore forming the model FEED (see Figure 1). This method also requires the establishment of the type of causality exerted between the variables. It is generally accepted that a positive (direct) causal link is noted with "S" or "+" and denotes a change in the same direction at both its ends, its reciprocal not being necessarily true. Meanwhile, an opposite fluctuation of the ending variables is considered a negative (indirect) causal link and is noted with "O" or "-". Again, the reciprocal is not necessarily true. In FEED, to facilitate understanding, all the positive influences (S-type) were drawn in green, while all the negative ones (O-type) were drawn in red. Another particularity of causal loops diagrams that also helps with the visualization and analysis of the outcome of the interconnections, is the type of causal loop. A reinforcing loop represents a cycle within which the same positive or negative variation is propagated and returned strengthened at the initial point. A balancing loop is one that breaks the cycle of the initially propagated variation and thus results in the decrease of its effect.

After the model was designed, we used TRUE (Temporal Reasoning Universal Elaboration) software created by Patrick Höuel. Not only was this software developed based on the work of Jay Forrester, but also was declared by Strasbourg University as a valuable modelling tool for analysis, simulation and optimization in various domains, including conflict and risk management, decision-making, macro- and micro-economic analysis, social mathematics etc. (www.true-world.com). This software enabled us to run the model forward and backward for a better interrogation of the system, seeking to understand its governing rules and sensitive points, 
thus approaching its validation, and also some practical answers related to food waste reinforcement.

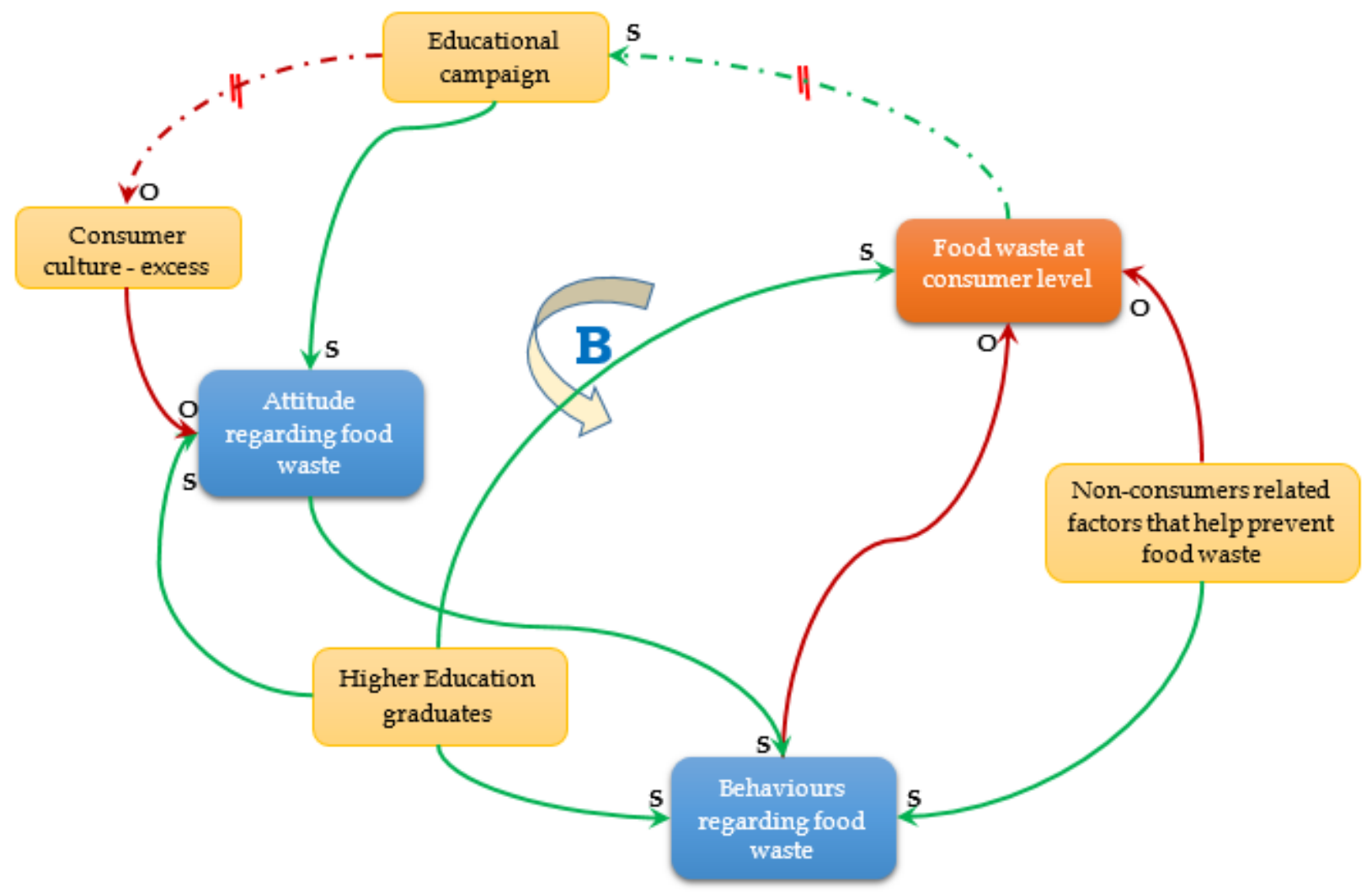

PICBE $\mid 760$

Figure 1. The FEED model

FEED starts from the idea that the number of higher education graduates, consumer culture, educational campaigns and all the non-consumer related factors that help prevent food waste have a major influence on food waste at the consumer level. Based on the literature review, it was established that an increase in the number of higher education graduates would lead to an increase in the amount of food wasted (Visschers et al., 2016; Secondi at al., 2015), as well as increases in the positive attitudes and behaviors regarding food waste (Toma et al., 2020; Qi \& Roe, 2016; Mallinson et al., 2016; Neff et al., 2015). The other influences were also established based on the previous literature review.

It is worth mentioning that FEED does not display any reinforcing loop so that to justify the imminence of a snowball effect working towards the destabilization of the system, herein meaning an inexorable growth in the amount of consumer food waste. On the contrary, the model shows at least one balancing loop (food waste - educational campaign - consumer culture - attitude regarding food waste - behavior regarding food waste - food waste again). According to its mission, this type of loop is meant to stabilize the system by resisting change, which is exactly what the above-mentioned loop does. This sounds like good news as it speaks about the absence of an already path dependence that would make it difficult to diminish food waste at consumer level, but the use of dynamic simulation will show whether this is actually true or not.

We chose to translate the model into the tenet of TRUE software by shaping each soft variable's fluctuation, this being necessary and sufficient according to Hanneman (1988), the 
introduction of precise parameters not being mandatory or of real gain for the accuracy of the forecasted evolution of the goal-variable (i.e., food waste).

As a first step, we decided the time spam for running the simulation at 30 years. Then it was necessary to set each variable's temporality, meaning setting their prioritization in computation. FEED considers the variables number of higher education graduates, consumer culture, educational campaign and all the non-consumer related factors that help prevent food waste as prerequisites that alter the state of attitudes and behaviors regarding food waste, therefore having the chronology set to " 1 ". Attitude regarding food waste is considered a cumulative variable which draws its manifestation based on consumer culture that unfolds (O-type) and the pressure of higher education graduates and educational campaign (S-type) and it is computed in the second step (i.e., its chronology is "2"). Behavior regarding food waste has its chronology set to " 3 " because it is obtained as an outcome of several causal influences: number of higher education graduates, attitude regarding food waste and non-consumer related factors (all of them with S-type connections). As a result, food waste at the consumer level for whose simulation the model is constructed will have the chronology set to " 4 ".

The next step involved the definition of each causality evolution shape and this was done by short programming sequences such as in the following example:

//"educational campaign"

$\mathrm{x}$, centre,pente is real

centre=p:repeat $/ 2$

$\mathrm{x}=$ nbt-centre

pente $=60$

$\mathrm{y}=1 /(1.2+\operatorname{Exp}(-\mathrm{x} /$ pente $))$

$\mathrm{y}=\operatorname{Round}(\mathrm{y}, 3)$

The code gives an ascending " $\mathrm{S}$ " sigmoid shape that stands for the idea of a gradual implementation of educational campaigns and therefore their impact over people's attitudes. However, in order to show that the variable refers to multiple campaigns, we went for sequencing this influence every 8 units of time during the entire time span. In a similar way, we set the evolution of consumer culture, respectively its influence over the time span on people's attitudes regarding food waste. However, in this case, the manifestation is continuous and the slope smoother. The same situation with the variables higher education graduates and non-consumer related factors, which have similar sequences of programming and therefore similar evolution shapes as shown in Figure 2. 

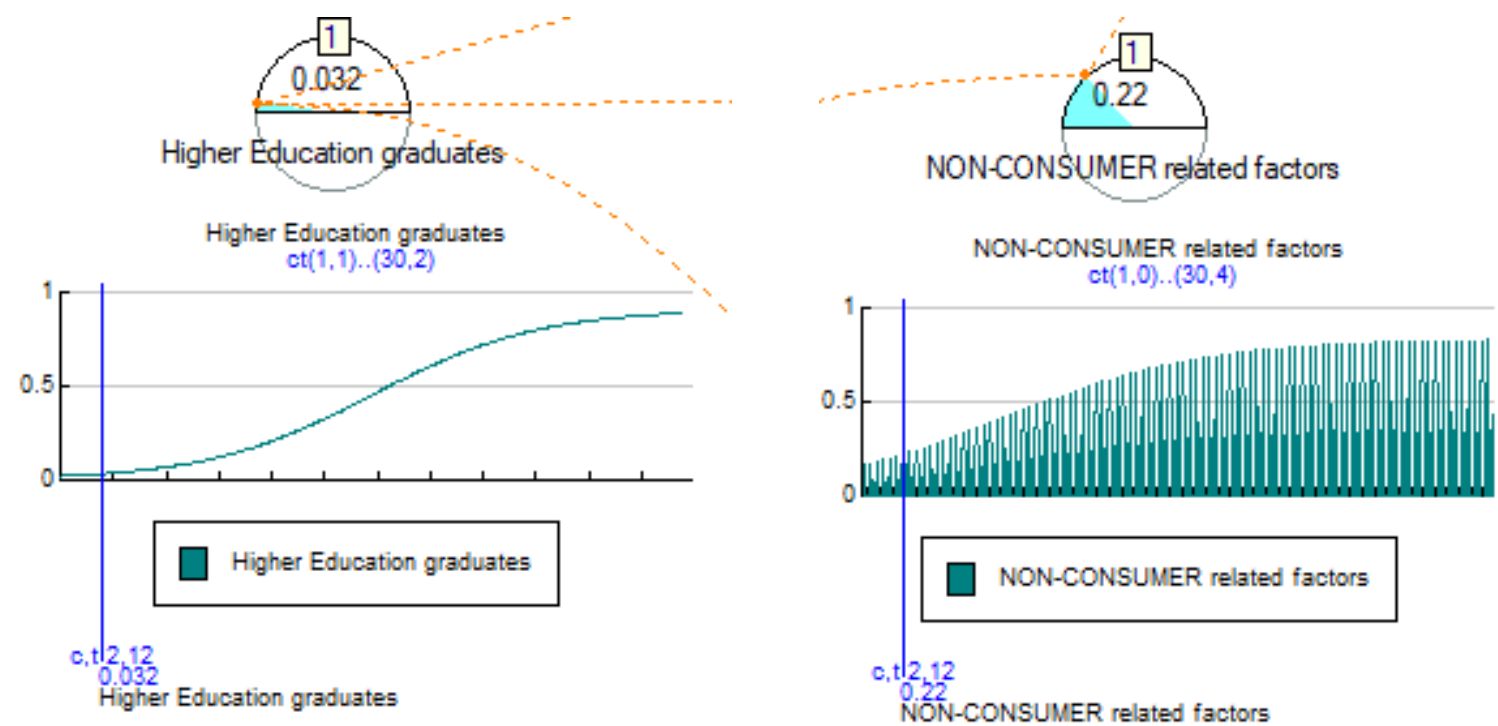

Figure 2. Snapshot of the evolution of: a. HE graduates; b. Non-consumer related factors

Out of the four above-mentioned variables, the former three alter the evolution of attitude regarding food waste, be it in a positive (i.e., education campaigns and higher education graduates) or a negative manner (i.e., consumer culture). Consequently, the shape of the cumulative variable evolution is obtained by the simple addition of their individual evolution curves:

//code for "ATTITUDE regarding FOOD WASTE"

$\mathrm{y}=$ Fvalue("educational campaign")

$\mathrm{y}+=$ Fvalue("Higher Education graduates")

$\mathrm{y}-=$ Fvalue("consumer-culture")

$\mathrm{y}=\operatorname{Round}(\mathrm{y}, 3)$

The result speaks about an ongoing rather negative attitude about food waste in the present and some positive break-ups until the $15^{\text {th }}$ year of the time span, when the variable has the value 0 . That is the moment when the attitude becomes mainly positive with only a few insertions of values that tend back to 0 (see Figure 3 ).

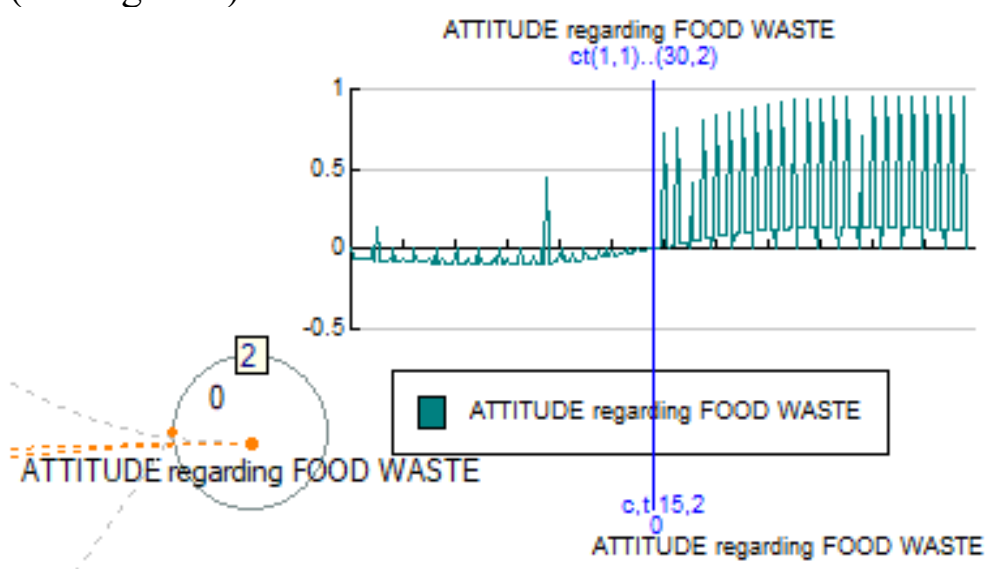

Figure 3. Evolution of the attitude regarding the food waste - snapshot

In the same vein, considering the multicausality that occurs in the meeting point behavior regarding food waste, the programming sequence included: //code for "BEHAVIOURS regarding FOOD WASTE" 
$\mathrm{y}=$ Fvalue("ATTITUDE regarding FOOD WASTE")

$\mathrm{y}+=$ Fvalue("Higher Education graduates")

$\mathrm{y}+=$ Fvalue("NON-CONSUMER related factors")

$\mathrm{y}=\operatorname{Round}(\mathrm{y}, 3)$

Thus, the evolution of this variable will register a faster or slower ascending/descending slope given the combination of the following headways: higher education graduates, non-consumer related factors, and attitude regarding food waste. As seen, most of the interconnections displayed by the model are ascending sigmoid influences, so the intuition of a sigmoid positive variation of the variable behaviors regarding food waste is justified and therefore confirmed by Figure 4 .

The evolution of the cumulative variable food waste at consumer level was obtained in a similar manner through the addition of the influence of higher education graduates and the subtraction of the influence of favorable behaviors and non-consumer related factors. The state of this threatening situation triggers the reiteration of some educational campaigns, although quite often delayed. In causal loop diagrams, this is considered a lag and it is indicated by a double slash crossing the connection of two adjoining flows (see Figure 1) and speaks about cause and effect that are not closely related in time and/or space. The model in TRUE also reflects this lag as follows:

//"educational campaign"

prs are currency

prs = Fvalue("FOOD WASTE at consumer level", False, nbt-3)

$\ldots$

IF $\operatorname{prs}>0$

$\mathrm{y}=\mathrm{y}+\mathrm{prs}$

ELSE

$\mathrm{y}=\mathrm{y}$

END

$\mathrm{y}=\operatorname{Round}(\mathrm{y}, 3)$

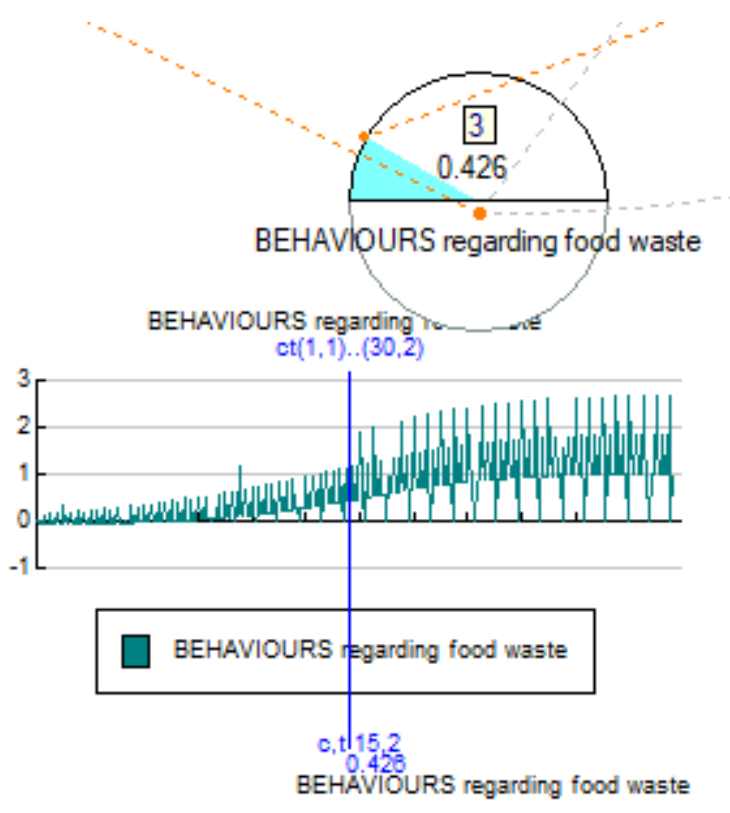

Figure 4. Evolution of behavior regarding food waste - Snapshot

After the inclusion of the above-mentioned causality, the evolution displayed by the flow educational campaign changed as reflected in Figure 5. 


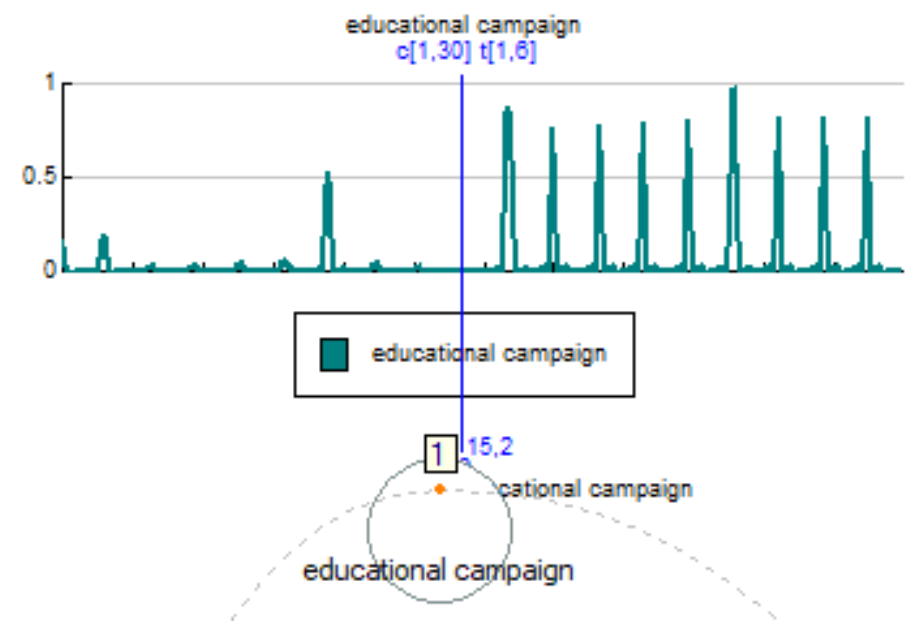

PICBE | 764

Figure 5. Evolution of educational campaign -Snapshot 2

Culture, whether organizational, national or economic, is based on individuals' emotions, perceptions, beliefs and assumptions irrespective of their root cause which, in time, turn into attitudes. Therefore, even if the general tendency is to consider educational campaigns entirely ineffective, they actually act as the water drops that shape the mountain. Consequently, we decided to connect the two flows from the model and the resulted fluctuation is displayed in Figure 6. stv are currency stv = Fvalue("educational campaign", False, nbt-1) $\mathrm{y}=\mathrm{y}$-stv

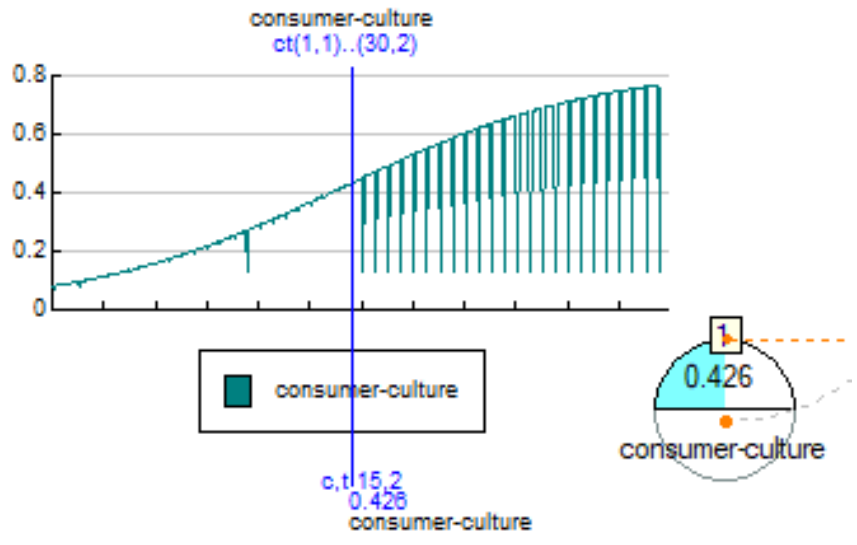

Figure 6. The evolution of consumer-culture - Snapshot 2 
The outcomes of these causalities on the goal-variable food waste at consumer level is shown in Figure 7. Somehow contradicting the intuition that made us expect a large increase in the amount of food wasted at consumer level, running the simulation reveals rather the opposite: an

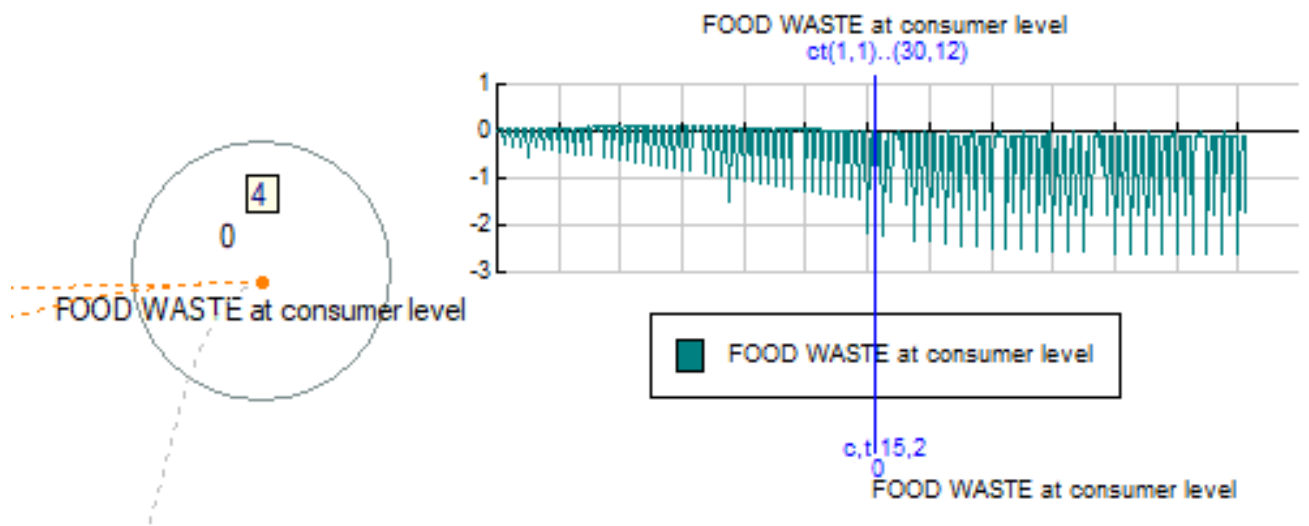

PICBE | 765

Figure 7. Evolution of food waste at consumer level - Snapshot

upcoming period characterized by a continuation of the already existent situation (more or less accentuated food waste), which starts changing with the $13^{\text {th }}$ year of the time span and displaying a decreasing slope that keeps the fluctuation of the variable under the 0 level.

\section{Research Results and Discussion}

Once FEED was completely transposed into the tenet of TRUE, we were able to run it forward and backward while changing some aspects related to its flows in order to investigate its evolution. Note that FEED does not consider any factors of influence over the number of higher education graduates since it is not a comprehensive model and it limited its scope to factors directly related to the relationship between education and food waste. Therefore, by adding some prerequisites for higher education graduates or other variables, we could face a different outcome of the goalvariable food waste, but that would be beyond the scope of the present paper.

What we can observe by running the simulation is that if we dissipate educational campaigns (i.e., decrease their incidence), the time span needed for the goal-variable to become negative enlarged (with one year in the case of a decrease of frequency of occurrence of the educational campaigns from once every 8 months to once every two years). If, in turn, we do the same with the non-consumer related factors, and we postpone its manifestation alongside that of educational campaigns, then the evolution maintains its shape, it only become smoother and enlarges a bit the time span necessary for the goal-variable to become negative (see Figure 8). 


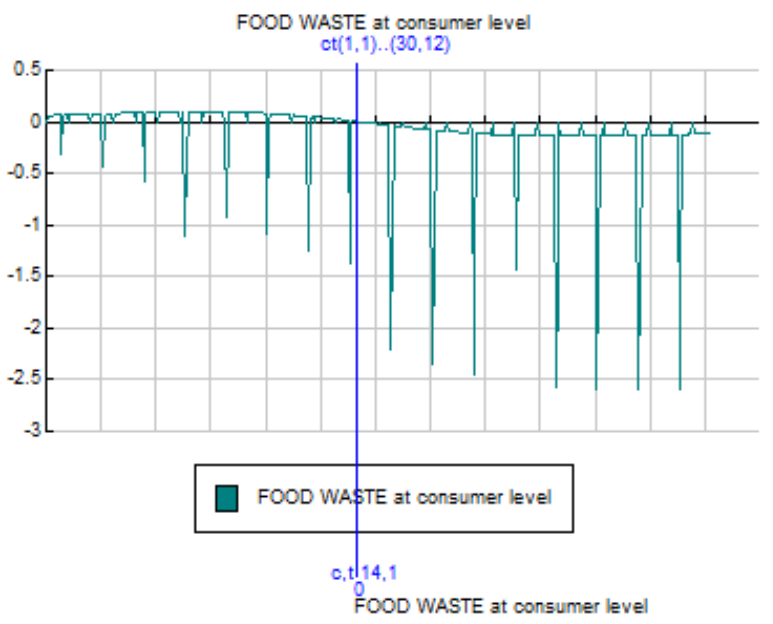

PICBE | 766

Figure 8. The evolution of food waste at consumer level after parameter changes

So far, the greatest benefit of our modeling project is to help the reader and/or decision makers see the importance of some actions (i.e., educational campaigns and non-consumer related factors) traditionally considered not powerful enough in comparison with trends such as the consumerist culture. FEED also highlights the need for a greater emphasis on the role of higher education systems, which should not only prepare students for the labor market, but also prepare students for the adoption of sustainable behaviors in their personal and professional lives.

\section{Conclusion}

The present study started from the intriguing direct influence of the number of HE graduates over consumer food waste, which was proven by several previous researches. This could induce the idea of a gradual but steady and inexorable increase of the amount of food wasted as the general tendency of mass education is to increase the number graduates of higher education. In order to find if everything is already lost as it may seem, we designed our model, FEED, by means of the causal loop diagrams method and then we translated into the tenet of a dynamic simulation software. There was no reinforcing loop displayed by FEED causal loop version, fact aligned with the evolution of our goal-variable when the simulation of the model was run. This makes us to suspect the possibility of some well-balanced human actions causing the fit and proper interinfluences evolution and thus enabling an under zero value of consumer food waste. But this conclusion merits future research.

We are confident that FEED represents a useful step in understanding the relationship between education and food waste as, in the end, the dynamic simulation of a model should trigger attention to details and a thorough analysis of the process itself rather than to the analysis of the results, as the former speeds learning and leads to better models and therefore better decisionmaking (Forrester, 1971; 1985). We hope that the model will help shed light of the intricacies of the connections between education and food waste and that future studies will pay closer attention to the impact of the level of education on factors that affect food waste at the consumer level. 


\section{References}

Abdelradi, F. (2018). Food waste behaviour at the household level: A conceptual framework. Waste Management, 71, 485-493.

Abeliotis, K., Lasaridi, K., Chroni, C. (2014). Attitudes and behaviour of Greek households regarding food waste prevention. Waste Management \& Research, 32(3), 237-240.

Aschemann-Witzel, J., De Hooge, I., Amani, P., Bech-Larsen, T., \& Oostindjer, M. (2015). Consumer-related food waste: Causes and potential for action. Sustainability, 7(6), 64576477.

Box, G. E. P., Draper, N. R. (1987). Empirical Model-Building and Response Surfaces, Wiley, London, p. 424.

Djekic, I., Miloradovic, Z., Djekic, S., \& Tomasevic, I. (2019). Household food waste in Serbia Attitudes, quantities and global warming potential. Journal of Cleaner Production, 229, 4452.

Forrester JW. (1961). Industrial Dynamics. Pegasus Communications: Waltham, MA.

Forrester, J.W. (1971/1985). The model versus a modelling process. System Dynamics Review 1(1): $133-134$.

Forrester, J.W., (1971b). World Dynamics. Pegasus Communications: Waltham, MA.

Forrester, J.W. (1999). System Dynamics: the Foundation Under Systems Thinking, Sloan School of Management, Massachusetts Institute of Technology, Cambridge, MA 02139, p.1

Gaiani, S., Caldeira, S., Adorno, V., Segrè, A., \& Vittuari, M. (2018). Food wasters: Profiling consumers' attitude to waste food in Italy. Waste Management, 72, 17-24.

Graham-Rowe, E., Jessop, D. C. \& Sparks, P. (2015). Predicting household food waste reduction using an extended theory of planned behaviour. Resources, Conservation and Recycling, 101, 194-202.

Hanneman, R.A. (1988). Computer-assisted theory building. Modeling dynamic social systems, Sage Publications

Hannon, B., Ruth, M. (2001). Dynamic Modeling, second edition, Springer-Verlag New York, Inc.

Karim Ghani, W. A., Rusli, I. F., Biak, D. R., \& Idris, A. (2013). An application of the theory of planned behaviour to study the influencing factors of participation in source separation of food waste. Waste Management, 33(5), 1276-1281.

Kim, D.H. (1993). A framework and methodology for linking individual and organizational learning: applications in TQM and product development, $\mathrm{PhD}$ thesis submitted in 1986 to the Sloan School of Management, Massachusetts Institute of Technology

Koivupuro, H.-K., Hartikainen, H., Silvennoinen, K., Katajajuuri, J.-M., Heikintalo, N., Reinikainen, A., \& Jalkanen, L. (2012). Influence of socio-demographical, behavioural and attitudinal factors on the amount of avoidable food waste generated in Finnish households. International Journal of Consumer Studies, 36(2), 183-191.

Lusk, J. L., \& Ellison, B. (2016). A note on modelling household food waste behaviour. Applied Economic Letters, 24(16), 1199-1202.

Mallinson, L. J., Russell, J. M., \& Barker, M. E. (2016). Attitudes and behaviour towards convenience food and food waste in the United Kingdom. Appetite, 103, 17-28.

Melbye, E. L., Onozaka, Y., \& Hansen, H. (2016). Throwing it all away: Exploring affluent consumers' attitudes toward wasting edible food. Journal of Food Products Marketing, 23(4), 416-429. 
Nässén, J. (2017). Anti-consumption beliefs among the Swedish general public. In C. Bala, \& W. Sculdzinski (Eds.) The $21^{\text {st }}$ Century Consumer, Proceedings of the International Conference on Consumer Research 2016 (pp. 173-188). Düsseldorf: Verbraucherzentrale NRW.

Neff, R. A., Spiker, M. L., \& Truant, P. L. (2015). Wasted food: U.S. consumers' reported awareness, attitudes, and behaviors. PLoS One, 10(6), e0127881.

PICBE $\mid 768$

Newton, P. (2013, April 18). "Green hypocrites? Behaviour change in a consumerist society". Retrieved from: theconversation.com/green-hypocrites-behaviour-change-in-aconsumerist-society-13002.

Pakpour, a. H., Zeidi, I. M., Emamjomeh, M. M., Asefzadeh, S., \& Pearson, H. (2014). Household waste behaviours among a community sample in Iran: An application of the theory of planned behaviour. Waste Management, 34(6), 980-986.

Porpino, G. (2016). Household food waste behavior: Avenues for future research. Journal of the Association for Consumer Research, 1(1), 41-51.

Qi, D., \& Roe, B. E. (2016). Household food waste: Multivariate regression and principal components analyses of awareness and attitudes among U.S. consumers. PLoS One, 11(7), e0159250.

Sadjadian, H., Taghirad, H.D. (2005). Comparison of different Methods for Computing the Forward Kinematics of a edundant Parallel Manipulator, Journal of Intelligent and Robotic Systems, Springer, 44(3), 225-246.

Secondi, L., Principato, L., \& Laureti, T. (2015). Household food waste behaviour in EU-27 countries: A multilevel analysis. Food Policy, 56, 25-40.

Senge, P.M. (1999), The fifth discipline. The art and practice of the learning organisation, Random House Business Books, London, Great Britain.

Stancu, V., Haugaard, P., \& Lähteenmäki, L. (2016). Determinants of consumer food waste behaviour: Two routes to food waste. Appetite, 96, 7-17.

Stefan, V., van Herpen, E., Tudoran, A. A., \& Lähteenmäki, L. (2013). Avoiding food waste by Romanian consumers: The importance of planning and shopping routines. Food Quality and Preference, 28(1), 375-381.

Sterman, J.D. (2002). All models are wrong: reflections on becoming a systems scientist, System Dynamics Review Vol. 18, No. 4, (Winter 2002): 501-531.

Toma, L., Font, M. C., \& Thompson, B. (2020). Impact of consumers' understanding of date labelling on food waste behaviour. Operational Research, 20, 543-560.

Visschers, V. H. M., Wickli, N., \& Siegrist, M. (2016). Sorting out food waste behaviour: A survey on the motivators and barriers of self-reported amounts of food waste in households. Journal of Environmental Psychology, 45, 66-78.

Yamakawa, H., Williams, I. D., Shaw, P. J., \& Watanabe, K. (2017). Food waste prevention: Lessons from the Love Food, Hate Waste campaign in the UK. In Proceedings Sardinia 2017 Sixteenth International Waste Management and Landfill Symposium. Cagliari: CISA Publisher.

Young, W., Russell, S. V., Robinson, C. A., \& Barkemeyer, R. (2017). Can social media be a tool for reducing consumers' food waste? A behaviour change experiment by a UK retailer. Resources, Conservation and Recycling, 117, 195-203. 